\title{
Expanding the Swiss autosomal marker set to 32 STRs
}

\author{
Martin Zieger ${ }^{1}\left[\mathbb{D}^{-} \cdot\right.$ Alexandre Gouy $^{2} \cdot$ Silvia Utz $^{1}$
}

Received: 4 May 2021 / Accepted: 24 May 2021 / Published online: 18 June 2021

(c) The Author(s) 2021

\begin{abstract}
By genotyping 1198 individuals with the Qiagen Investigator ${ }^{\circledR}$ HDplex Kit, we expand the Swiss autosomal STR dataset to 32 loci, providing additional resources for complex kinship cases. We present the first high-quality allele frequency dataset for loci D2S1360, D5S2500, D7S1517, and D10S2325 that will be accessible through the ENFSI reference database STRidER. For loci D3S1744, D4S2366, D6S474, D8S1132, and D21S2055, we provide a first European STRidER dataset.
\end{abstract}

Keywords Switzerland $\cdot$ STR $\cdot$ Investigator HDplex $\cdot$ Kinship $\cdot$ Population data

The Qiagen Investigator ${ }^{\circledR}$ HDplex Kit provides valuable additional information to assess complex kinship scenarios, by supplementing the set of forensic standard short tandem repeat (STR) loci [1]. We genotyped 1198 Swiss individuals from a previous population study for those nine additional loci.

DNA extractions were prepared as described [2]. Multiplex PCR was performed in a reduced reaction volume of $12.5 \mu \mathrm{L}$. Capillary electrophoresis was run on a 3500x1 genetic analyzer (ThermoFisher, USA) and data interpretation carried out with Genemapper ${ }^{\circledR}$ ID-X, v1.4 (Thermo Fisher, US). Details on the population structure can be found in [2]. Allele frequencies, forensic parameters, and test for Hardy-Weinberg equilibrium (HWE) with 10,000 permutations were calculated using STRAF [3], excluding two genotypes with three-allelic patterns. Results are listed in Table S1. None of the nine additional loci shows significant deviation from HWE. Allele frequencies are also available as Familias [4, 5] input file for all 32 loci (Table S2). Pairwise

Martin Zieger

Martin.Zieger@irm.unibe.ch

Alexandre Gouy

alexandre.gouy@protonmail.com

Silvia Utz

Silvia.Utz@irm.unibe.ch

1 Institute of Forensic Medicine, Forensic Molecular Biology Department, University of Bern, Sulgenauweg 40, 3007 Bern, Switzerland

2 Gouy Data Consulting, Sentier de Renges 4A, 1026 Denges, Switzerland
$\mathrm{F}_{\mathrm{ST}}$ values for the six regional sub-populations defined in [2] revealed no intra-national differences, as expected (Table S3).

All genotype data for the three loci D12S391, D18S51, and SE33 are concordant with the data previously generated with Promega PowerPlex® Fusion 6C [2]. One sample showed a triplet in D6S474, another one in D10S2325. Five samples showed an almost complete allele dropout in marker D2S1360. This partial dropout has previously been observed and has been shown to be due to a SNP in the primer binding site [6]. Interestingly, in our dataset, all partial dropouts concerned allele 21 , suggesting an association in the studied population of this allele with the SNP in the flanking region. We also detected a couple of off ladder alleles. All variants are listed in Table S4.

For marker D21S2055, heterozygote balance was usually below $60 \%$ if one allele consisted of less than 30 repeats and the other one of more than 30 repeats, limiting the utility of this marker for trace analysis and particularly DNA mixtures. However, there is no clear-cut allele length boundary for this phenomenon. We also observed pronounced peak imbalance between a couple of other pairs of alleles, e.g., $17.1 / 29$ or $31 / 33$. A similar observation has already been made by Tillmar et al. [7]. This suggests an association of a structural difference in the flanking regions with the STR repeat length, which might be worth a future investigation by sequencing.

Expanding the STR dataset increases the number of syntenic loci. Since ignoring linkage between loci might have an impact on certain kinship scenarios [8], we list the information for relevant syntenic loci, including loci distances 
based on Phillips et al. [9], in Table S5. We checked for linkage disequilibrium with the genepop $\mathrm{R}$ package $[10,11]$ by performing an exact test with 50,000 iterations and 1000 batches. For the complete dataset of 32 loci, we could not detect any significant linkage disequilibrium after Bonferroni correction (see Table S6).

The present dataset has passed the quality control for STRidER [12] and obtained the STRidER accession number STR000368.

Supplementary Information The online version contains supplementary material available at https://doi.org/10.1007/s00414-021-02624-w.

Acknowledgements We thank all the donors for participating in the project and Ina Krebber (Interregionale Blutspende SRK) for her help in organizing the sample collection. We thank especially Ludmilla Lieder, but also Sina Mani, Xenia Merz, Nurdan Tura, and Adrian Zurbrügg for technical assistance and Alexandra Haas as well as Colin Tièche for critical reading of the manuscript.

Funding Open Access funding provided by Universität Bern.

\section{Declarations}

Samples are the same as in [2]. All samples were collected with informed written consent. They were reversibly anonymized, to permit the donors to exert their right to withdraw their sample at any time. The Institute of Forensic Medicine, University of Bern, obtained the samples under an arbitrary number. The written consent documents with the names of the donors remained with the Red Cross. All documents distributed to the donors upon sampling were submitted to the responsible cantonal ethical committee and approval obtained.

Conflict of interest The authors declare no competing interests.

Open Access This article is licensed under a Creative Commons Attribution 4.0 International License, which permits use, sharing, adaptation, distribution and reproduction in any medium or format, as long as you give appropriate credit to the original author(s) and the source, provide a link to the Creative Commons licence, and indicate if changes were made. The images or other third party material in this article are included in the article's Creative Commons licence, unless indicated otherwise in a credit line to the material. If material is not included in the article's Creative Commons licence and your intended use is not permitted by statutory regulation or exceeds the permitted use, you will need to obtain permission directly from the copyright holder. To view a copy of this licence, visit http://creativecommons.org/licenses/by/4.0/.

\section{References}

1. Tillmar AO, Mostad P (2014) Choosing supplementary markers in forensic casework. Forensic Sci Int Genet 13:128-133

2. Zieger M, Utz S (2019) A "forensic biobank" to establish comprehensive genetic frequency data for Switzerland. Forensic Sci Int Genet 40:46-51

3. Gouy A, Zieger M (2017) STRAF-a convenient online tool for STR data evaluation in forensic genetics. Forensic Sci Int Genet 30:148-151

4. Egeland T, Mostad PF, Mevag B, Stenersen M (2000) Beyond traditional paternity and identification cases selecting the most probable pedigree. Forensic Sci Int 110(1):47-59

5. Kling D, Tillmar AO, Egeland T (2014) Familias 3 - Extensions and new functionality. Forensic Sci Int Genet 13:121-127

6. Inokuchi S, Yamashita Y, Nishimura K, Nakanishi H, Saito K (2017) Identification of a common single nucleotide polymorphism at the primer binding site of D2S1360 that causes heterozygote peak imbalance when using the Investigator HDplex Kit. Int J Legal Med 131(6):1531-1535

7. Tillmar AO, Nilsson H, Kling D, Montelius K (2013) Analysis of Investigator HDplex markers in Swedish and Somali populations. Forensic Sci Int Genet 7(1):e21-e22

8. Nothnagel M, Schmidtke J, Krawczak M (2010) Potentials and limits of pairwise kinship analysis using autosomal short tandem repeat loci. Int J Legal Med 124(3):205-215

9. Phillips C, Ballard D, Gill P, Court DS, Carracedo A, Lareu MV (2012) The recombination landscape around forensic STRs: accurate measurement of genetic distances between syntenic STR pairs using HapMap high density SNP data. Forensic Sci Int Genet 6(3):354-365

10. Raymond M, Rousset F (1995) Genepop (Version-1.2) - population-genetics software for exact tests and ecumenicism. J Hered 86(3):248-249

11. Rousset F (2008) genepop'007: a complete re-implementation of the genepop software for Windows and Linux. Mol Ecol Resour 8(1):103-106

12. Bodner M, Bastisch I, Butler JM, Fimmers R, Gill P, Gusmao L, Morling N, Phillips C, Prinz M, Schneider PM, Parson W (2016) Recommendations of the DNA Commission of the International Society for Forensic Genetics (ISFG) on quality control of autosomal Short Tandem Repeat allele frequency databasing (STRidER). Forensic Sci Int Genet 24:97-102

Publisher's note Springer Nature remains neutral with regard to jurisdictional claims in published maps and institutional affiliations. 